\title{
False appearance of bilateral pneumothorax in a patient with hypoplastic left lung
}

\author{
Murat Ersin Cardak, MD, ${ }^{\mathrm{a}}$ Mehmet Tukel, MD, ${ }^{\mathrm{a}}$ Recep Demirhan, MD, ${ }^{\mathrm{a}}$ and \\ Hasan Fevzi Batırel, MD, PhD, , ${ }^{\text {,b }}$ Istanbul, Turkey
}

Hypoplasia of the lung is a congenital or acquired condition that is characterized by a decrease in the size, number, or both of vascular, alveolar, and bronchial elements. ${ }^{1}$ This developmental abnormality can lead to unusual clinical signs. Most patients described in the literature are newborns and infants. ${ }^{1,2}$ Chronic infections in childhood can also cause lung hypoplasia. We present a patient with left lung hypoplasia and right lung hyperinflation that was diagnosed after a condition mimicking bilateral pneumothorax.

\section{CLINICAL SUMMARY}

A 17-year-old girl was admitted to the emergency department with shortness of breath. She was tachypneic and tachycardic with bilateral decreased breath sounds and desaturation $(85 \%)$ while receiving $8 \mathrm{~L} / \mathrm{min}$ oxygen. Chest radiographic analysis showed bilateral pneumothorax and mild scoliosis (Figure 1, A). Bilateral chest tubes were placed. Although pneumothorax resolved after chest tube

From the Thoracic Surgery Clinic, ${ }^{a}$ Dr Lutfi Kirdar Kartal Training and Research Hospital, and the Department of Thoracic Surgery, ${ }^{\mathrm{b}}$ Marmara University Faculty of Medicine, Istanbul, Turkey.

Disclosures: Authors have nothing to disclose with regard to commercial support.

Received for publication Aug 12, 2011; revisions received Oct 13, 2011; accepted for publication Oct 31, 2011; available ahead of print Dec 8, 2011.

Address for reprints: Hasan Fevzi Batirel, MD, PhD, Ministry of Health of TurkeyMarmara University Hospital, Thoracic Surgery Department, 7th Floor, F wing, Fevzi Cakmak Mah, Mimar Sinan Cad, No: 41, 34899, Ust Kaynarca, Pendik, Istanbul, Turkey (E-mail: hbatirel@marmara.edu.tr).

J Thorac Cardiovasc Surg 2012;143:e19-e20

$0022-5223 / \$ 36.00$

Copyright (C) 2012 by The American Association for Thoracic Surgery doi:10.1016/j.jtcvs.2011.10.085 placement, a chest computed tomographic (CT) scan was performed to rule out any additional pathology because pneumothorax coincided with her menstruation. The CT scan showed a hypoplastic left lung with cystic bronchiectasis and a hyperexpanded horseshoe-shaped right lung that occupied the whole chest cavity (Figure 1,B). Several blebs and emphysematous areas were seen at the apex of the right lung. She had a history of severe pneumonia caused by measles in infancy. A quantitative pulmonary perfusion scan showed $21 \%$ perfusion to the left side. Echocardiographic results were normal, there was no evidence for pulmonary hypertension, and a thoracic CT angiogram showed normal vascular anatomy. Her prior medical history also included frequent episodes of pulmonary infection. On bronchoscopic examination, her left bronchus was of relatively normal size; however, she had purulent secretions. The patient was then intubated with a single-lumen endotracheal tube. After left sixth interspace thoracotomy, we found that the right lung was covered by the mediastinal pleural envelope and occupied almost all of the left chest cavity. The left lung was very small, with fully developed anatomic structures; however, it had several abscess cavities, and there were a few inflammatory adhesions. All the hilar structures, including the left pulmonary artery, were displaced inferiorly in the chest cavity. The left pulmonary artery was tiny (approximately $1 \mathrm{~cm}$ in diameter).

We first performed a left pneumonectomy followed by a right upper lobe wedge resection to the scar area in the right apical segment of the lung, where there was obvious air leak, and the patient's own pleura was used to buttress the staple line. No pleurodesis was performed. The patient recovered quickly after the operation and was discharged

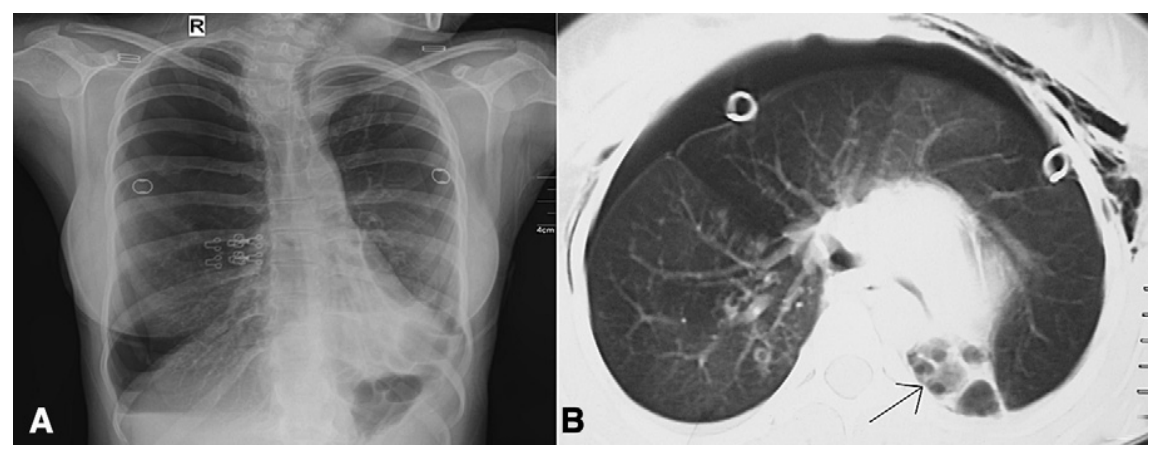

FIGURE 1. A, Chest radiograph showing bilateral pneumothorax and air-fluid level in the right lower zone. The left chest cavity is smaller than the right, and there is mild scoliosis. B, Two chest tubes can be seen in both sides of the thorax but within the right pleural envelope. The left lung is very small and located at the posteromedial area of the left lower chest cavity (arrow). 
TABLE 1. Features of horseshoe lung in infants and lung hypoplasia in adults

\begin{tabular}{lll}
\hline & \multicolumn{1}{c}{ Infant } & \multicolumn{1}{c}{ Adult } \\
\hline Symptoms & Dyspnea, wheezing & Frequent infections in childhood and adolescence, hemoptysis \\
Pathophysiology & - Associated with congenital anomalies (usually right lung & • Chronic inflammatory disease \\
& hypoplasia and anomalous pulmonary venous return to the & • No connection between the 2 lungs (separate pleural cavities) \\
& vena cava in $80 \%$ [Scimitar syndrome]) & • Usually expansion of upper lobe and anterior mediastinal \\
& - Fusion of right and left lower lobes in the posterior & herniation \\
& mediastinum & - Hypoplastic but normal vascular anatomy \\
& - Small or no pulmonary artery, associated pulmonary & $\bullet$ Associated mild scoliosis \\
Workup & hypertension & CT scan, echocardiography \\
Treatment & Pulmonary CT angiography, MRI, echocardiography & Surgical resection \\
Prognosis & Symptomatic treatment & Good long-term survival \\
\hline
\end{tabular}

$C T$, Computed tomography; $M R I$, magnetic resonance imaging.

on the fourth postoperative day. Pathology revealed chronic inflammation and bronchiectasis in the left lung and blebs and emphysema in the right lung. She is alive and well at 18 months with a forced vital capacity of $2.29 \mathrm{~L}(85 \%)$, a forced expiratory volume in 1 second of $1.14 \mathrm{~L}(50 \%)$, and a diffusing capacity of the lung for carbon monoxide of $23.2 \mathrm{~mL}$ of $\mathrm{CO} \cdot \mathrm{min}^{-1} \cdot \mathrm{mm} \mathrm{Hg}^{-1}(113 \%)$.

\section{DISCUSSION}

Hypoplasia of the lung is a congenital or acquired anomaly in which the gross morphology of the lung is essentially unremarkable other than a size anomaly. Developmental anomalies of the lung can result in complete absence of one lung (agenesis), rudimentary bronchus, and no lung tissue (aplasia) or underdevelopment of the lung with normal structural components (hypoplasia).

Congenital cases are usually diagnosed during early childhood and are generally associated with Scimitar syndrome. ${ }^{2}$ A similar congenital condition called horseshoe lung (posterior mediastinal fusion of the lower lobes) can be diagnosed during infancy or adulthood. ${ }^{1,2}$ Hypoplasia of one lung and compensatory hyperinflation of the contralateral lung is usually the result of chronic inflammatory lung disease causing pulmonary hypoplasia., ${ }^{3,4}$ It is generally characterized by herniation of the anterior segment of the right upper lobe to the upper half of the left hemithorax. The features of these conditions are shown in Table 1.
Only 2 cases have been reported in the literature similar to our patient. ${ }^{3,4}$ In both cases the left lung was hypoplastic. However, the clinical presentation with pneumothorax is the only such case in the literature. We did not perform pleurodesis so as not to obliterate the single functional pleural cavity.

In our patient hypoplasia of the left lung was secondary to measles-related pneumonia during early infancy. Pneumonectomized children show similar adaptive hyperinflation of the remaining lung, which is due to the growth capacity of the remaining lung with increases in the number of alveoli. ${ }^{5}$ At least half of the alveoli regenerates, and neovascularization contributes to restoration of gas exchange area.

The occurrence of pneumothorax in this giant hyperinflated lung mimicked a bilateral pneumothorax, and this was a unique observation in a very uncommon clinical condition.

\section{References}

1. Lutterman J, Jedeikin R, Cleveland DC. Horseshoe lung with left lung hypoplasia and critical pulmonary venous stenosis. Ann Thorac Surg. 2004;77:1085-7.

2. McDonald ES, Hartman TE. A rare case of horseshoe lung presenting in adulthood and associated with a pulmonary sling: case report and review of the literature. J Thorac Imaging. 2010;25:W97-9.

3. Sinha PK, Misra S, Unnikrishnan M, Bhuyan RR. Horseshoe lung secondary to hypoplastic left lung for pneumonectomy. J Cardiothorac Vasc Anesth. 2007;21: 250-2.

4. Ciric I, Meyers MP, Mayba J, Anthonisen NR. Autopneumonectomy with compensatory lung growth. Can Respir J. 2003;10:271-3.

5. Eren S, Eren MN, Balci AE. Pneumonectomy in children for destroyed lung and the long-term consequences. J Thorac Cardiovasc Surg. 2003;126:574-81. 